\title{
Electrospun Gelatin Membrane Cross-Linked by a Bis(diarylcarbene) for Oil/Water Separation: A New Strategy To Prepare Porous Organic Polymers
}

\author{
Xi Yu, ${ }^{\dagger}$ Pengfei Yang, ${ }^{*}{ }^{\dagger}$ Mark G. Moloney, ${ }^{\ddagger}{ }^{\circ}$ Liang Wang, ${ }^{\S}$ Jinku Xu, ${ }^{\dagger}$ Yongqing Wang, ${ }^{\dagger}$ Lian Liu, \\ and Yunlin Pan ${ }^{\dagger}$ \\ ${ }^{\dagger}$ School of Chemistry and Pharmaceutical Engineering, Qilu University of Technology (Shandong Academy of Sciences), Jinan \\ 250353, PR China \\ ${ }^{\ddagger}$ Chemistry Research Laboratory, Department of Chemistry, University of Oxford, Oxford OX1 3TA, U.K. \\ ${ }^{\S}$ College of Chemistry and Pharmaceutical Sciences, Qingdao Agricultural University, Qingdao 266109, PR China
}

Supporting Information

ABSTRACT: Porous organic polymers (POPs) as absorbing materials have attracted increasing attention. Here, we report a new approach to prepare these polymers for selective oil absorption from oil/water mixtures. Perfluoroalkylbis(diaryldiazomethane) was synthesized and used to modify the surface of an electrospun gelatin membrane by a carbene insertion reaction, not only to immobilize the porous network morphology by cross-linking but also to introduce perfluoroalkyl groups for oil/water separation. The membrane was characterized to show its surface and bulk properties, as well as its performance for absorption capacity, selectivity, and

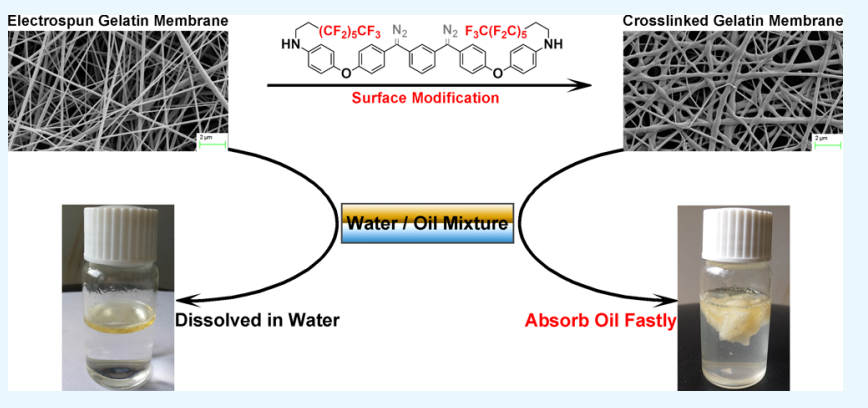
renewability. This approach offers a new horizon in the preparation of POPs for oil/water separation.

\section{INTRODUCTION}

Absorbent materials have attracted much attention because of their wide range of applications in catalysis, ${ }^{1,2}$ sensors, ${ }^{3,4}$ gas storage $^{5}$ and separation, ${ }^{6}$ drug delivery, ${ }^{7,8}$ and oil/water separation., 10 In particular, materials for selective oil absorption are gaining more and more attention on account of marine oil spills, ${ }^{11}$ which due to increasing environmental pollution is now a global issue. ${ }^{12,13}$ Unlike traditional remediation pathways which have serious limitations, ${ }^{14,15}$ selective absorbent materials would not only clean the water but also allow the possibility to recycle the oil and generate an economic return. ${ }^{16,17}$ There is one kind of oil/water separation material that has the ability to selectively absorb water, which offers the possibility for the demulsification of oil, and which is an economic and efficient way to prepare and purify the crude oil. $^{18,19}$ However, the preparation of these water-absorbent nanocomposite materials is usually complicated and costly, and this along with their inferior performance has limited their applications on the industrial scale.

Nowadays, many porous organic polymers (POPs) such as covalent organic frameworks, ${ }^{20}$ conjugated microporous polymers, ${ }^{21}$ hypercross-linked polymers, ${ }^{22}$ polymers of intrinsic microporosity, ${ }^{23}$ and porous aromatic frameworks ${ }^{24}$ have been used as absorbent materials because of their lightweight and versatility. However, there are still two severe shortcomings: First, most POPs with diverse well-defined nanomorphology cannot be easily achieved because the introduction of cross- linkers or grafted templates limits the processability of the resulting polymers. Second, it is difficult to prevent changes to the desired nanomorphology. As a result, great efforts have been made to fabricate nanomorphology-persistent POPs via various cross-linking strategies. ${ }^{25}$

Electrospinning is a simple and versatile technique for the construction of polymers with expanded surface area and is achieved by fabricating a continuous and nonwoven netlike structure of nanofibers. ${ }^{26,27}$ Recently, Liu et al..$^{28}$ developed an approach to prepare electrospun nanocomposites with a high surface area and controlled macroscopic shape. Using this approach, we fabricate a porous structure of the gelatin nanofiber membrane via electrospinning and the subsequent introduction of carbene insertion modification gives a structure with cross-linked gelatin fibers, in which the fiber morphology is maintained along with the newly endowed hydrophobicity. This provides a new way to prepare absorbent materials for oil/ water separation and further expands the application of carbene chemistry in materials, which have already been used for the introduction of color, ${ }^{29,30}$ hydrophobicity, ${ }^{31,32}$ antibacterial activity, ${ }^{33,34}$ fluorescence, ${ }^{35}$ and payload delivery. ${ }^{36,37}$

Received: January 25, 2018

Accepted: March 28, 2018

Published: April 9, 2018 


\section{RESULTS AND DISCUSSION}

2.1. Synthesis of Diazomethane and Its Reactivity. As shown in Scheme 1, 4-phenoxyaniline was reacted with

Scheme 1. Synthesis of the Precursor and Modification of the Gelatin Membrane ${ }^{a}$

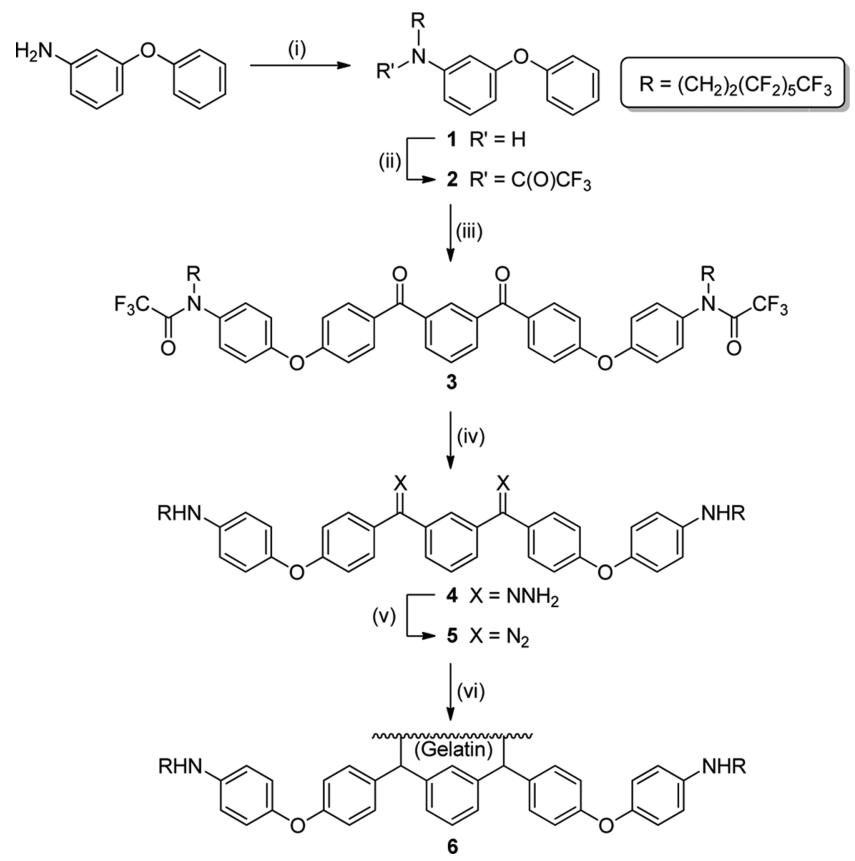

${ }^{a}$ Conditions: (i) $\mathrm{I}\left(\mathrm{CH}_{2}\right)_{2} \mathrm{C}_{6} \mathrm{~F}_{13}, 140{ }^{\circ} \mathrm{C}, 12 \mathrm{~h},(48 \%)$; (ii) TFAA, pyridine, DCM, $5{ }^{\circ} \mathrm{C}$ to $\mathrm{rt}$, overnight (95\%); (iii) isophthaloyl chloride, $\mathrm{AlCl}_{3}$, DCM, $5{ }^{\circ} \mathrm{C}$ to rt, $10 \mathrm{~h}$ (80\%); (iv) $\mathrm{NH}_{2} \mathrm{NH}_{2}, \mathrm{H}_{2} \mathrm{O}$, $\mathrm{HOAc}, \mathrm{EtOH}$, reflux, $45 \mathrm{~h}$ (90\%); (v) $\mathrm{MnO}_{2}, \mathrm{Na}_{2} \mathrm{SO}_{4}, \mathrm{KOH}, \mathrm{DCM}$, rt, $24 \mathrm{~h}$ (92\%); and (vi) UV light.

$1 H, 1 H, 2 H, 2 H$-perfluorooctyl iodide to give compound $\mathbf{1}$, which was subsequently reacted with trifluoroacetic anhydride (TFAA) to give amide 2 , and converted to ketone 3 by Friedel-Crafts reaction. ${ }^{38}$ The ketone group of 3 was treated with hydrazine monohydrate to give hydrazone 4 , which was oxidized with $\mathrm{MnO}_{2}$ to give bis(diaryldiazomethane) 5. The reactivity of diazomethane 5 was assessed by treatment with acetic acid and compared with the reported diazomethanes, before being used for modification. In Figure 1, clear changes of UV spectra at $528 \mathrm{~nm}$ appeared after acetic acid was added and

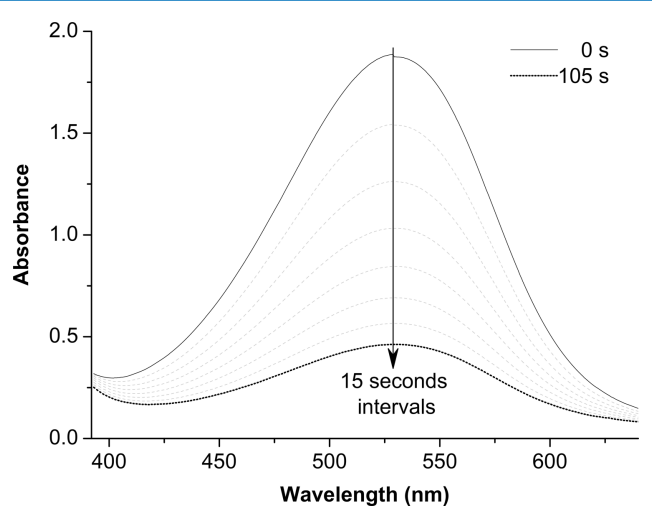

Figure 1. UV spectra for the reaction of diazomethane 5 with acetic acid at different reaction times. this peak was suitable to calculate the reaction rate. According to the method in the literature, the reaction rate of diazomethane 5 was determined to be $1.34 \times 10^{-2} \mathrm{~s}^{-1}$, which was very similar to the nonfluoro diazomethane. ${ }^{33}$ This demonstrated that diazomethane could be easily converted into carbenes under UV light ${ }^{39}$ and efficiently reacted with gelatins to introduce perfluoroalkyl groups onto the surface. In addition, different reactivities between diazomethane 5 and reported fluoro-diazomethanes ${ }^{31}$ are noteworthy, which might be attributed to the separation of the diazo group and the perfluoroalkyl group.

2.2. Preparation and Modification of Gelatin Membranes. Generally, there are three main advantages of electrospun nanofibrous membranes of the gelatin as a liquid carrier. First, its fibrous morphology gives a large surface area that is necessary for liquid absorption. Second, abundant hydrogen bonds among polymer chains lead to effective and efficient absorption of polar liquids. Third, it is easy for the gelatin to be obtained from waste or food by-products. However, there is one serious disadvantage which limits its application for oil absorption; it is strongly hydrophilic so that it has poor oil selectivity. Therefore, a surface modification of the gelatin membrane was introduced according to the idea of "phase-selective organogelators", 9,40,41 which had a hydrophobic surface to prevent water from entering and a lot of hydrogen bonds to expand the oil-absorbing capacity. That is, the surface modification of the gelatin membrane is required to (i) make it sufficiently hydrophobic to avoid water absorption and immobilize the selectively absorbed oil and (ii) maintain its porous morphology and hydrogen bond structure to give a large oil absorption capacity among the fibrillar network. To meet these two requirements, the gelatin membrane was fabricated via electrospinning, followed by its modification and cross-linking with perfluoroalkyldiazomethanes.

As shown in Figure 2(1), the electrospun gelatin membrane showed an interconnected and continuous morphology with a nanofibrous structure. The membrane was immersed into a solution of diazomethane 5 in dichloromethane (DCM) and dried under nitrogen flow. The gelatin was completely wetted but did not dissolve at all in DCM, which was very important because the membrane would maintain its original structure during the loading process of diazomethanes. The loaded membrane was then irradiated under UV light to generate carbenes and accomplish the modification through the $\mathrm{X}-\mathrm{H}$ (X $=\mathrm{C}$ and $\mathrm{N}$ ) insertion of carbene onto the surface. The modified membrane showed a unique network morphology, which is shown in Figure 2(2).

2.2.1. XPS Analysis. As for the chemical composition on the surface of the gelatin, the modified membrane should be very different from the pure membrane and X-ray photoelectron spectroscopy (XPS) analysis was carried out to explore these differences. Compared with those in Figure 3(1), the characteristic peaks of fluorine (F 1s at $688.60 \mathrm{eV}$ ) in Figure 3(2) confirmed the existence of perfluoroalkyl groups on the surface of modified gelatin. Moreover, the percentage of nitrogen was lowered on the surface of the modified gelatin because the modification layer derived from diazomethane $\mathbf{5}$ covered the amino acid residues of the gelatin that contain high levels of nitrogen. Figure $3(3,4)$ shows the C 1s spectra of gelatin membranes before and after modification, from which the peaks of $\mathrm{CF}_{2}(291.55 \mathrm{eV})$ and $\mathrm{CF}_{3}(293.90 \mathrm{eV})$ were clearly found. These two peaks also proved that the gelatin had been successfully modified. In addition, the peak area ratio of $\mathrm{CF}_{2}$ to 

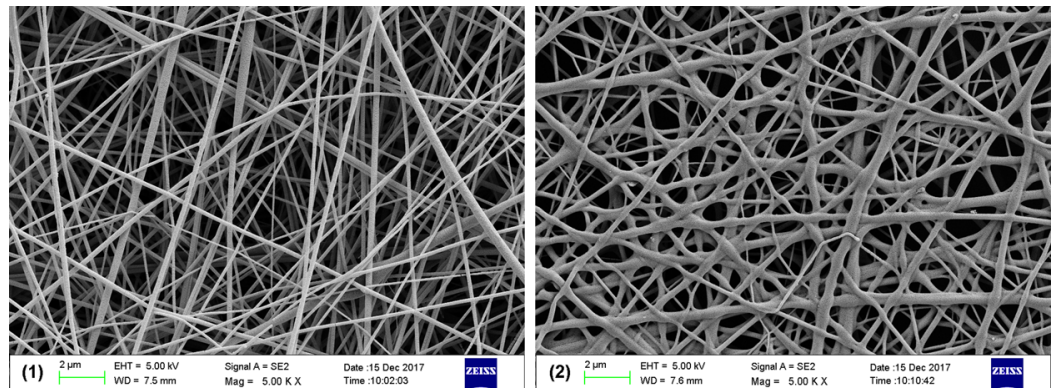

Figure 2. SEM pictures of electrospun gelatin membranes: (1) pure gelatin and (2) modified gelatin.
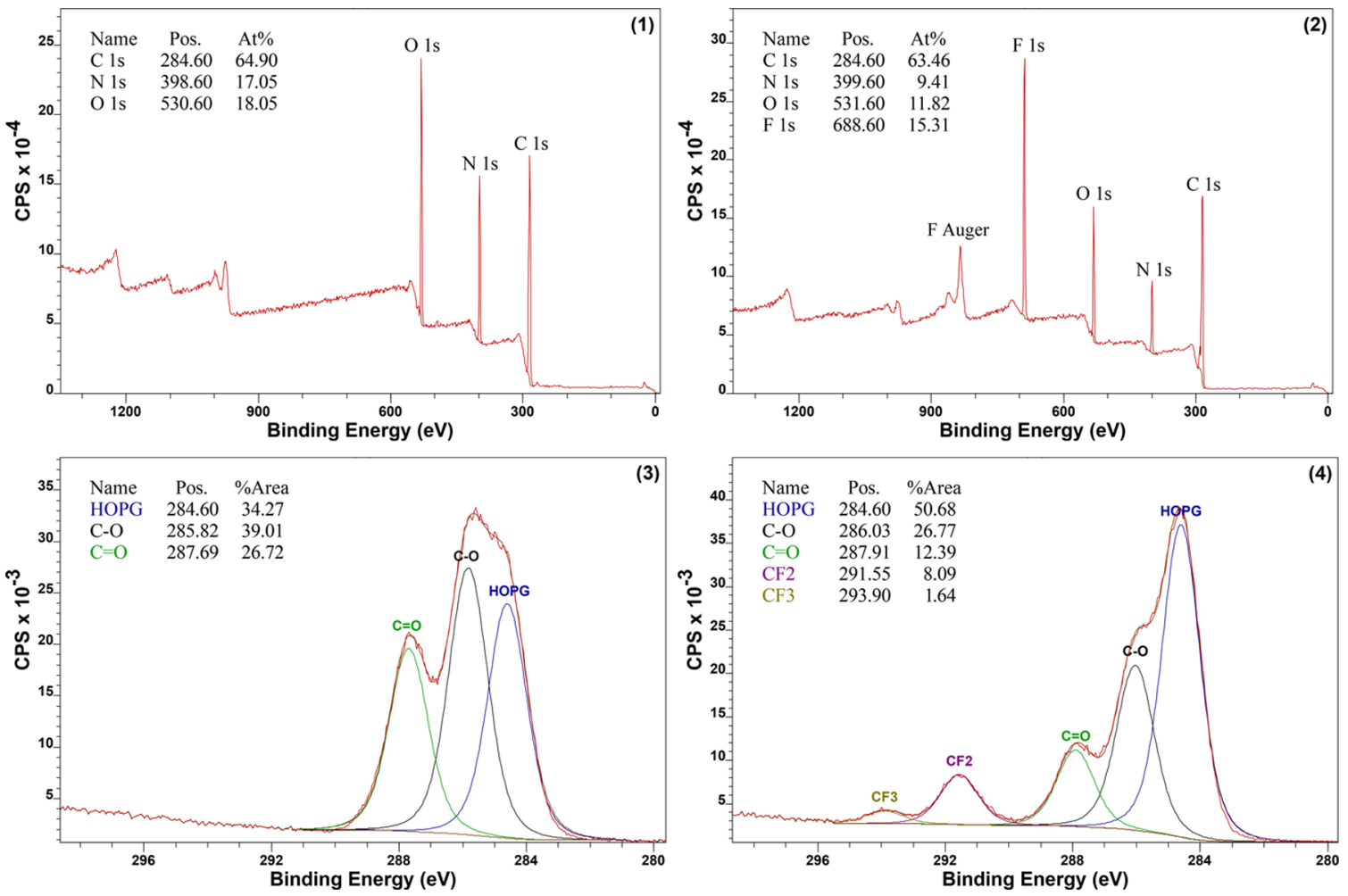

Figure 3. XPS spectra of pure gelatin and modified gelatin: (1) survey of pure gelatin; (2) survey of modified gelatin; (3) C 1s spectra of pure gelatin; and (4) C 1s spectra of modified gelatin.

$\mathrm{CF}_{3}$ was 4.93 , which was in accordance with the chemical structure of perfluoroalkyl groups.

2.2.2. Contact Angle Analysis. Water contact angle was measured to evaluate the wetting property of the modified surface. Pure gelatin is hydrophilic (water contact angle, $57^{\circ}$ ) and may even dissolved by water drops if the test time is prolonged. However, the water contact angle of modified gelatin membrane 6 was increased to $132^{\circ}$, demonstrating the presence of the perfluoroalkyl groups which have a low surface free energy. The contact angle of peanut oil $\left(\gamma=2.87 \times 10^{-2} \mathrm{~N}\right.$ $\mathrm{m}^{-1}$ ) was also measured for the modified gelatin membrane, which was determined to be lipophilic with a contact angle of $35^{\circ}$. These data proved that the modified gelatin membrane was suitable for oil absorption because of its hydrophobicity and lipophilicity. Moreover, a further treatment by diazomethane cross-linked the gelatin membrane and made it insoluble in water, which was very different from the pure gelatin.

2.2.3. Pore Area and Pore Size. The surface area of gelatin before and after modification was tested by the Brunauer-
Emmett-Teller (BET) method, which was found to be varied slightly from 3.82 to $3.45 \mathrm{~m}^{2} \mathrm{~g}^{-1}$. This result confirmed that the porous structure via electrospinning was maintained after modification. However, BET method is normally just suitable for nanosized porous materials. For the nanofibrous gelatin containing micrometer pores, mercury porosimeter method should also be conducted to characterize the surface property, such as total pore area, porosity, and pore size distribution. It was determined that total pore area varied from 6.65 to $2.06 \mathrm{~m}^{2}$ $\mathrm{g}^{-1}$ and porosity varied from 81.9 to $56.1 \%$ after modification, which indicated that parts of the initial macropores might disappear. The curve of pore size distribution is shown in Figure 4. There are two peak regions for the pure gelatin membrane. One is very sharp with the peak value of $831 \mathrm{~nm}$, and the other is a broad peak region of $5 \times 10^{3}$ to $6 \times 10^{4} \mathrm{~nm}$. After modification, the sharp peak shifted to $1323 \mathrm{~nm}$ and the broad peak region almost disappeared, which was in accordance with the reduction of total pore area and porosity.

2.2.4. Tensile Test. Because the backbone of the gelatin membrane is hydrophilic and its surface is hydrophobic, any 


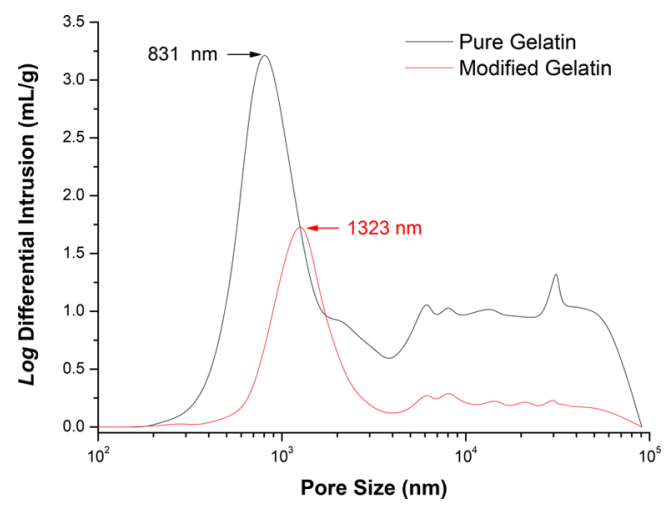

Figure 4. Curve of pore size distribution before and after modification.

crack on the surface or exposure of backbones might do harm to its oil absorption. Therefore, the tensile test via a static mechanical analyzer was used to characterize its mechanical property. The stress-strain curve of the gelatin membrane at room temperature (rt) is shown in Figure 5, which illustrated a

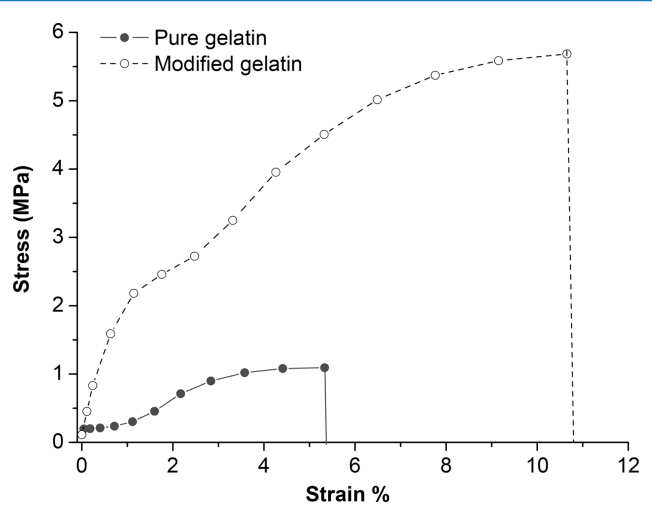

Figure 5. Stress-strain curve of pure gelatin and modified gelatin.

large difference after modification and cross-linking. The tensile strength of the pure gelatin electrospun membrane was very low, which indicated hard polymer behavior. However, after being treated with diazomethanes, the gelatin membrane showed higher tensile modulus and bigger elongation at break. That is, the treatment of the membrane with diazomethanes enhanced the bending strength and fracture toughness simultaneously, so as to guarantee a long service life for oil absorption.

2.3. Application of Oil/Water Separation. As known to all, the pure gelatin membrane is very loose and easy to be dissolved in water. When it was added to the peanut oil/water mixture, the membrane was dissolved completely after being shaken for sometime and the peanut oil was clearly seen as the upper liquid in Figure 6(1). In contrast to that phenomenon, the modified gelatin membrane was not dissolved in the oil/ water mixture. After being shaken, the membrane was still floated on the partly emulsive mixture (Figure 6(2)). The upper oil layer was then completely absorbed into the membrane only after 3 min (Figure 6(3)).

2.3.1. Absorption Capacity and Universality. Using the mixtures of water with various organic liquids such as methanol, glycol, dimethyl sulfoxide, peanut oil, petrol, and diesel, the application of the gelatin membrane was investigated so as to expand the universality of this selective absorption. As shown in Figure 7 , the membrane could absorb a wide range of organic

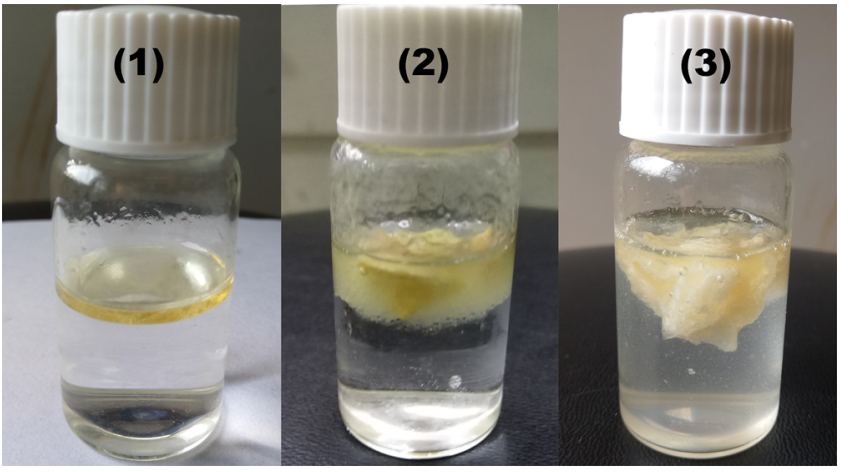

Figure 6. Photos of selective oil absorption: (1) pure gelatin membrane in the oil/water mixture; (2) modified gelatin membrane in the oil/water mixture, $0 \mathrm{~min}$; and (3) modified gelatin membrane in the oil/water mixture, $3 \mathrm{~min}$.

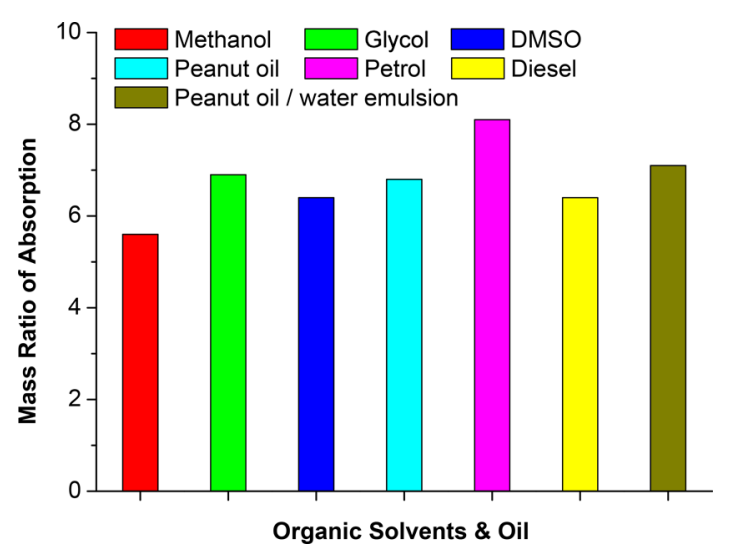

Figure 7. Mass ratio of the absorption of various solvents and oil in water.

liquids with rather large capacity (5.6-8.1 times larger than its own weight), indicating its distinguish advantages in oil/water separation. This data could be compared with some common absorbing materials, such as polystyrene fibers (7.1 times), ${ }^{42}$ polypropylenes (7-10 times), ${ }^{43}$ silane-coated polyurethane (PU) sponges (10-12 times), ${ }^{44}$ and $\mathrm{Fe} / \mathrm{C}$ nanocomposites (4.5-7.5 times). ${ }^{45}$ Moreover, some more expensive materials might have better absorbing property, such as carbon nanotubecoated sponges (15-25 times), ${ }^{46}$ graphene oxide-modified PU foams (26-41 times), ${ }^{47}$ and boron nitride nanosheets (20-33 times) ${ }^{48}$ It is noteworthy that the oil/water/surfactant mixture is likely to form an emulsion that makes the oil separation more difficult. By contrast, when the gelatin membrane was put into the emulsion of water/oil/TWEEN 80 (9:1:1, mass ratio), it still absorbed the peanut oil effectively, which was very useful for the real field application.

2.3.2. Absorption Selectivity and Water Repellency. It was noteworthy that when organic liquids were selectively absorbed from the oil/water mixtures, the water absorption of the membrane should be fully blocked to enhance the absorption selectivity. To determine its selectivity, the absorbed gelatin membrane with peanut oil was extracted with cyclohexane; the oil was easily extracted into the cyclohexane layer and purified by rotary evaporation under vacuum. According to that, the selectivity of oil absorption, which was named recovery efficiency, was determined to be $98 \%$. This data could be compared with some typical absorbing materials, such as filter paper $(91 \%),{ }^{49}$ cotton fabric $(97.8 \%),{ }^{50}$ and graphene oxide- 
modified PU foams (93.8\%). ${ }^{47}$ Moreover, long-time water repellency was measured by immersing the membrane into water and weighing at regular intervals. According to the comparison of the increased mass to the original mass, the percentage of water absorption was determined and shown in Figure 8. It was found that the membrane absorbed a little

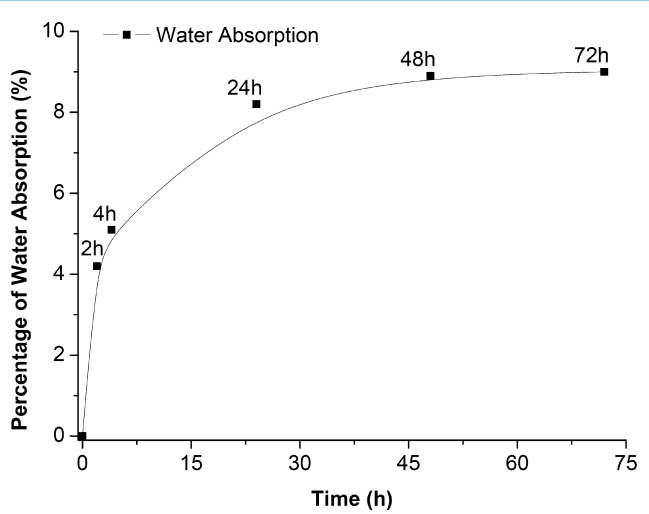

Figure 8. Water absorption of the gelatin membrane over time.

water after one day, and then the absorption rate slowed down and ultimately held the value of $9.0 \%$. Even so, the absorption rate of oil was much faster than that of water, which indicated excellent absorption selectivity.

2.3.3. Recovery Ability and Gelatin Leakage. It is very important for the gelatin membrane to be reused efficiently during the real application. Therefore, the gelatin membrane was recycled five times, and the recovery efficiency of each circulation was measured; the results are shown in Figure 9. It

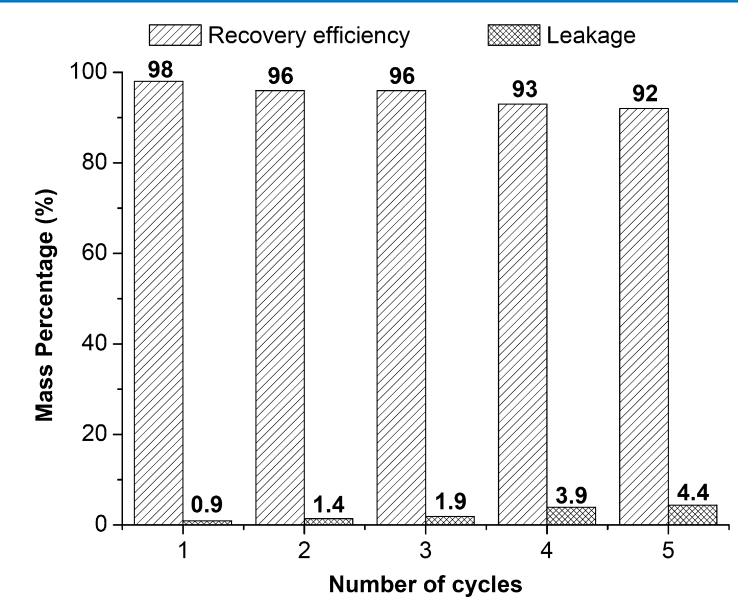

Figure 9. Recovery ability and gelatin leakage of the membrane after recycling.

was found that although the recovery efficiency of the membrane gradually lowered with the number of cycles, the material still showed good absorbing ability (92-98\%) after five times recycling. Moreover, the leakage of gelatin was another important factor to evaluate the recovery ability. It was shown in Figure 9 that the leakage of gelatin increased from 0.9 to $4.4 \%$ after five times recycling, which showed good recovery ability for oil/water separation.

\section{CONCLUSIONS}

Gelatin was fabricated by electrospinning, modified, and crosslinked by a perfluoroalkyldiazomethane through a carbene insertion reaction. This approach gave the gelatin membrane with a porous morphology and large surface area and with hydrophobicity and lipophilicity properties, which were proved by scanning electron microscopy (SEM), XPS, BET, mercury porosimeter, contact angle test, and tensile test. This unique structure made the electrospun gelatin membrane suitable for selective oil absorption and immobilization. The oil uptake ratio and recovery test showed that the membrane had good absorption capacity, selectivity, and recovery ability. This discovery established a general way to prepare POPs with hydrophobicity and lipophilicity, which were expected to have wide applications on oil/water separation.

\section{EXPERIMENTAL PART}

4.1. Materials and Characterization. Gelatin was purchased from Aladdin Co. with the grade of biochemical reagent. The peanut oil $\left(\gamma=2.87 \times 10^{-2} \mathrm{~N} \mathrm{~m}^{-1}\right)$ was supplied by Shandong Luhua Group Co., Ltd. Diesel oil (0\#) was purchased from PetroChina Co., Ltd. The other reagents (A.R.) were purchased from Sinopharm Chemical Reagent Co., Ltd. All of the chemicals were used as received.

NMR spectra were recorded by a AVANCE III spectrometer, and attenuated total reflection-infrared spectra were recorded by a Tensor 27 spectrometer, both of which were manufactured by Bruker Co. UV spectra were collected with a Shimadzu UV2600 spectrometer.

SEM photographs were taken on a SUPRA 55 thermal field emission SEM (Zeiss Co., Germany) after the samples were coated with gold ( $\sim 20 \mathrm{~nm}$ thickness).

XPS analysis was performed with a VG-Escalab X-ray Photoelectron Spectrometer VGX900 using Al K $\alpha$ radiation with an energy of $1486.6 \mathrm{eV}$ at an operating pressure less than 1 $\times 10^{-8} \mathrm{mBar}$. The pass energy was set at $50 \mathrm{eV}$ for the survey scan of the sample and $20 \mathrm{eV}$ for scans of specific areas: $\mathrm{C} 1 \mathrm{~s}, \mathrm{~N}$ $1 \mathrm{~s}, \mathrm{O} 1 \mathrm{~s}$, and F 1s. CasaXPS peak fitting software was used to analyze the spectra, and a reference charge correction of 284.5 $\mathrm{eV}$ for $\mathrm{C} 1 \mathrm{~s}$ was used. The area under the elemental peaks and standard sensitivity factors (C, 1.00; O, 2.93; N, 1.80; and F, 4.43) were used to calculate relative chemical composition within the surface and near-surface sampling depth of the XPS.

Static contact angle was measured using a DSA-100 contact angle instrument manufactured by Krüss Co. with a $1 \mu \mathrm{L}$ water drop size. The BET surface area analysis was performed by nitrogen absorption experiments using a Micromeritics Tristar II PLUS analyzer. The pore size and its distribution were measured with a PM-60 mercury porosimeter. Tensile testing was performed on a Testometric AX universal strength testing machine at rt with a sample size of $50 \mathrm{~mm} \times 5 \mathrm{~mm}$.

4.2. Synthesis of Bis(aryldiazomethane)s. 4.2.1. 4Phenoxy- $\mathrm{N}-(1 \mathrm{H}, 1 \mathrm{H}, 2 \mathrm{H}, 2 \mathrm{H}$-perfluorooctyl)aniline (1). $1 \mathrm{H}, 1 \mathrm{H}, 2 \mathrm{H}, 2 \mathrm{H}$-Perfluorooctyl iodide $(4.74 \mathrm{~g}, 10.0 \mathrm{mmol})$ and 4-phenoxyaniline $(7.40 \mathrm{~g}, 40.0 \mathrm{mmol})$ were added into a 100 $\mathrm{mL}$ flask. The mixture was heated to $140{ }^{\circ} \mathrm{C}$ and stirred for 12 h. The crude product was purified by chromatography on a silica gel column eluting with petrol ether/ethyl acetate $(1: 10)$ to give product $\mathbf{1 b}$ as a yellow liquid $(48 \%) . \delta \mathrm{H}(400.3 \mathrm{MHz}$, $\left.\mathrm{CDCl}_{3}\right): 2.22(\mathrm{t}, 2 \mathrm{H}), 3.37(\mathrm{~m}, 3 \mathrm{H}), 6.84-7.41(\mathrm{~m}, 9 \mathrm{H}) . \delta \mathrm{C}$ $\left(100.1 \mathrm{MHz}, \mathrm{CDCl}_{3}\right): 30.44,36.39,114.01,117.30,121.37$, 
122.20, 129.60, 143.44, 148.47, 158.90. HRMS $m / z:[\mathrm{M}+\mathrm{H}]^{+}$ calcd for $\mathrm{C}_{20} \mathrm{H}_{15} \mathrm{ONF}_{13}, 532.09406$; found, 532.09409.

4.2.2. 2,2,2-Trifluoro-N-(4-phenoxyphenyl)-N$(1 \mathrm{H}, 1 \mathrm{H}, 2 \mathrm{H}, 2 \mathrm{H}$-perfluorooctyl)acetamide (2). 1 (2.00 g, 3.76 $\mathrm{mmol})$ and pyridine $(0.89 \mathrm{~mL}, 11.3 \mathrm{mmol})$ were dissolved in DCM $(20 \mathrm{~mL})$ and cooled down to $5{ }^{\circ} \mathrm{C}$ in an ice/water bath. A solution of TFAA $(0.79 \mathrm{~mL}, 5.65 \mathrm{mmol})$ in DCM $(5 \mathrm{~mL})$ was added dropwise to the flask. The mixture was then heated to rt and stirred overnight. After that, the mixture was washed with water $(50 \mathrm{~mL} \times 3)$, dried with $\mathrm{MgSO}_{4}$, and concentrated under vacuum to acquire a light yellow liquid (95\%). $\delta \mathrm{H}(400.3$ $\left.\mathrm{MHz} \mathrm{CDCl}_{3}\right): 2.48(\mathrm{t}, 2 \mathrm{H}), 4.04(\mathrm{t}, 2 \mathrm{H}), 7.06-7.45(\mathrm{~m}, 9 \mathrm{H})$. $\delta \mathrm{C}\left(100.1 \mathrm{MHz}, \mathrm{CDCl}_{3}\right): 28.10,44.59,118.72,119.95,124.54$, 129.51, 130.09, 132.77, 155.72, 158.68. HRMS $m / z:[\mathrm{M}+\mathrm{H}]^{+}$ calcd for $\mathrm{C}_{22} \mathrm{H}_{14} \mathrm{O}_{2} \mathrm{NF}_{16}, 628.07636$; found, 628.07649.

4.2.3. $N, N^{\prime}-(($ Isophthaloylbis(4,1-phenylene))bis(oxy))bis(4, 1 -phenylene))bis(2,2,2-trifluoro- $\mathrm{N}-(1 \mathrm{H}, 1 \mathrm{H}, 2 \mathrm{H}, 2 \mathrm{H}$ perfluorooctyl)acetamide (3). Aluminum chloride (0.949 g, $7.06 \mathrm{mmol})$ and $2(1.50 \mathrm{~g}, 2.82 \mathrm{mmol})$ were added to DCM $(20 \mathrm{~mL})$, and the solution was cooled to $5{ }^{\circ} \mathrm{C}$ with an ice/water bath. A solution of isophthaloyl chloride $(0.287 \mathrm{~g}, 1.41 \mathrm{mmol})$ in DCM $(5 \mathrm{~mL})$ was added dropwise to the flask. After the addition was finished, the ice/water bath was moved away and the reaction mixture was stirred for $10 \mathrm{~h}$. Then, the mixture was washed with $\mathrm{HCl}$ aq. solution $(2 \mathrm{M}, 50 \mathrm{~mL} \times 3)$, dried with $\mathrm{MgSO}_{4}$, and concentrated under vacuum. The crude product was purified by chromatography on a silica gel column eluting with petrol ether/ethyl acetate $(5: 1)$ to give product 3 as a yellow solid (80\%). Mp 64-68 ${ }^{\circ} \mathrm{C}$; $\delta \mathrm{H}\left(400.3 \mathrm{MHz}, \mathrm{CDCl}_{3}\right)$ : $2.51(\mathrm{t}, 4 \mathrm{H}), 4.07(\mathrm{t}, 4 \mathrm{H}), 7.14-7.21(\mathrm{~m}, 18 \mathrm{H}) . \delta \mathrm{C}(100.1$ $\left.\mathrm{MHz}, \mathrm{CDCl}_{3}\right): 28.21,44.69,118.36,120.48,129.93,132.66$, $134.56,137.97,156.75,160.46,194.41 . \mathrm{HRMS} m / z:[\mathrm{M}+\mathrm{Na}]^{+}$ calcd for $\mathrm{C}_{52} \mathrm{H}_{28} \mathrm{O}_{6} \mathrm{~N}_{2} \mathrm{~F}_{32} \mathrm{Na}$, 1407.13286; found, 1407.13369.

4.2.4. 4, $4^{\prime}-\left(\left(\left(1 E, 1^{\prime} E\right)-1,3-P h e n y l e n e b\right.\right.$ is (hydrazonomethylene))bis(4,1-phenylene))bis(oxy))bis( $N$ $(1 \mathrm{H}, 1 \mathrm{H}, 2 \mathrm{H}, 2 \mathrm{H}$-perfluorooctyl)aniline (4). A suspension of 3 $(0.50 \mathrm{~g}, 0.36 \mathrm{mmol})$ in ethanol $(35 \mathrm{~mL})$ was treated with hydrazine monohydrate $(0.35 \mathrm{~mL}, 7.22 \mathrm{mmol})$. Two drops of acetic acid were added as a catalyst. The resulting mixture was heated to a gentle reflux for $45 \mathrm{~h}$. After being cooled to rt, the solution was evaporated under vacuum. DCM $(50 \mathrm{~mL})$ was added to the residue, and the resulting solution was washed with $\mathrm{NaCl}$ aq. solution $(0.9 \mathrm{M}, 50 \mathrm{~mL} \times 3)$, dried with $\mathrm{MgSO}_{4}$, and concentrated under vacuum to acquire a light yellow solid (90\%). The crude product 4 as a mixture of diastereoisomers was used without further purification. $\mathrm{Mp} 110-114{ }^{\circ} \mathrm{C} . \delta \mathrm{H}$ $\left(400.3 \mathrm{MHz}, \mathrm{CDCl}_{3}\right): 2.30(\mathrm{t}, 4 \mathrm{H}), 3.41(\mathrm{t}, 4 \mathrm{H}), 3.55(\mathrm{~s}, 2 \mathrm{H})$, 6.84-8.41 (m, 18H), $7.02(\mathrm{~s}, 4 \mathrm{H}) . \delta \mathrm{C}\left(100.1 \mathrm{MHz}, \mathrm{CDCl}_{3}\right)$ : $30.67,36.39,113.94,113.99,117.51,117.61,121.82,127.81$, 130.30, 144.01, 147.50, 148.76, 159.43. HRMS $m / z:[\mathrm{M}+\mathrm{H}]^{+}$ calcd for $\mathrm{C}_{48} \mathrm{H}_{35} \mathrm{O}_{2} \mathrm{~N}_{6} \mathrm{~F}_{26}, 1221.24008$; found, 1221.23900.

4.2.5. 4,4'-((1,3-Phenylenebis(diazomethylene))bis(4,1phenylene))bis(oxy))bis $(\mathrm{N}-(1 \mathrm{H}, 1 \mathrm{H}, 2 \mathrm{H}, 2 \mathrm{H}$-perfluorooctyl)aniline (5). 4 (0.22 g, $0.18 \mathrm{mmol})$ was dissolved in DCM (10 $\mathrm{mL})$ in a $100 \mathrm{~mL}$ flask. A mixture of manganese dioxide (81 $\mathrm{mg}, 0.94 \mathrm{mmol})$, sodium sulfate $(72 \mathrm{mg}, 0.5 \mathrm{mmol})$, and potassium hydroxide $(22 \mathrm{mg}, 0.50 \mathrm{mmol})$ was added to the flask and stirred for $24 \mathrm{~h}$ in the dark at rt. Then, the mixture was filtered through a pad of Celite. The filtrate was concentrated under vacuum to yield a purple solid (92\%) and then stored at subambient temperature to avoid decomposition. Mp $126{ }^{\circ} \mathrm{C}$ (decomposed). $\delta \mathrm{H}(400.3 \mathrm{MHz}$, $\left.\mathrm{CDCl}_{3}\right): 2.43(\mathrm{t}, 4 \mathrm{H}), 3.59(\mathrm{t}, 4 \mathrm{H}), 3.76(\mathrm{~s}, 2 \mathrm{H}), 6.84-8.41(\mathrm{~m}$,
$18 \mathrm{H}), \delta \mathrm{C}\left(100.1 \mathrm{MHz}, \mathrm{CDCl}_{3}\right): 31.16,61.22,113.49,117.61$, $117.64,120.60,126.91,143.07,147.69,156.92$.

4.3. Kinetic Studies. A series of diazomethane 5 solutions in DCM $\left(2.0 \times 10^{-2}, 1.5 \times 10^{-2}, 1.0 \times 10^{-2}, 5.0 \times 10^{-3}\right.$, and $\left.1.0 \times 10^{-2} \mathrm{~mol} \mathrm{~L}^{-1}\right)$ were prepared to obtain UV spectra. The relationship between UV absorbance and concentration was recorded and fitted linearly to calculate the molar absorption coefficient. This allowed a calibration curve from absorbance to concentration to be determined, which was necessary for kinetic studies. Then, to the cuvette with a diazomethane $\mathbf{5}$ solution $\left(1.3 \times 10^{-2} \mathrm{~mol} \mathrm{~L}^{-1}\right)$ were added two drops of acetic acid (about 20 times excess). The mixture was immediately scanned by the UV spectrometer at regular intervals $(15 \mathrm{~s})$ until adequate spectra were recorded for the calculation of the reaction rate $\left(25^{\circ} \mathrm{C}\right.$, in $\left.\mathrm{CH}_{2} \mathrm{Cl}_{2}\right)$.

4.4. Preparation of Gelatin Nanofibrous Membranes. Gelatin $(8.0 \mathrm{~g})$ was dissolved in a solution $(42 \mathrm{~g})$ of acetic acid/ water $(9: 1, \mathrm{v} / \mathrm{v})$ at $\mathrm{rt}$ to give a light yellow liquid. The solution was added into a syringe and pumped to a self-made electrostatic spinning machine with a flow rate of $5 \mathrm{~mL} \mathrm{~h}^{-1}$. The voltage was $20 \mathrm{kV}$, and the collection distance was $10 \mathrm{~cm}$. The nanofiber was collected on the surface of an aluminum foil to form a gelatin membrane, which was subsequently stored in a vacuum drier. After that, a solution $\left(0.05 \mathrm{~g} \mathrm{~mL}^{-1}, 5\right.$ drops $\approx$ $0.25 \mathrm{~mL}$ ) of 5 in DCM was dropped onto the surface of the electrospun gelatin membrane $(50 \mathrm{~mm} \times 50 \mathrm{~mm} \times 2 \mathrm{~mm})$. After the solution was evaporated at $\mathrm{rt}$ in the dark, the gelatin fiber was covered with diazomethane. The mixture was then irradiated under UV light for $10 \mathrm{~min}$ until the color of the membrane changed from pink to orange. The mixture was filtered, and the solid was washed by DCM five times and dried to yield the amino-modified gelatin membrane 6 .

4.5. Determination of the Ratio of Uptake. Typically, a piece of cross-linked gelatin membrane was dropped into organic solvents or oil and stayed for $3 \mathrm{~min}$ to achieve absorption equilibrium. The ratio of uptake $q_{\mathrm{m}}$ was calculated from eq 1 .

$$
q_{\mathrm{m}}=\frac{m_{1}-m_{0}}{m_{0}}
$$

where $m_{0}$ is the mass of gelatin before absorption and $m_{1}$ is the mass of absorbed gelatin.

4.6. Determination of Recovery Efficiency. The absorbed gelatin was extracted with cyclohexane $(20 \mathrm{~mL} \times$ 3) under an ultrasonic oscillator. The organic solution was combined, concentrated under vacuum, and dried in an oven $\left(80^{\circ} \mathrm{C}\right)$ to obtain the recycled oil. The percentage of recovery efficiency $r_{\mathrm{m}}$ was calculated from eq 2 . The solid was directly dried in an oven $\left(80{ }^{\circ} \mathrm{C}\right)$ and used for another circulation, which was run five times.

$$
r_{\mathrm{m}}=\frac{m_{2}}{m_{1}-m_{0}} \times 100 \%
$$

where $m_{0}$ is the mass of gelatin before absorption, $m_{1}$ is the mass of absorbed gelatin, and $m_{2}$ is the mass of recovered oil.

4.7. Determination of Gelatin Leakage. As shown in part 4.6, the gelatin membrane was used for oil absorption and the similar circulation was run five times. After each circulation, the final weight of the dried gelatin was measured, which was compared with its initial weight. Therefore, the percentage of leakage $l_{\mathrm{m}}$ was calculated from eq 3 . 


$$
l_{\mathrm{m}}=\frac{m_{n-1}^{\prime}-m_{n}^{\prime}}{m_{n-1}^{\prime}} \times 100 \%
$$

where $m_{n-1}^{\prime}$ is the initial mass of gelatin before the $n$th circulation and $m_{n}^{\prime}$ is the final mass of gelatin after the $n$th circulation.

\section{ASSOCIATED CONTENT}

\section{S Supporting Information}

The Supporting Information is available free of charge on the ACS Publications website at DOI: 10.1021/acsomega.8b00162.

$$
\text { Spectra of all compounds (PDF) }
$$

\section{AUTHOR INFORMATION}

\section{Corresponding Author}

*E-mail: ypf@qlu.edu.cn (P.Y.).

\section{ORCID $\odot$}

Pengfei Yang: 0000-0003-4346-7367

Mark G. Moloney: 0000-0002-4440-3632

\section{Notes}

The authors declare no competing financial interest.

\section{ACKNOWLEDGMENTS}

This work is financially supported by the National Natural Science Foundation of China (nos. 21406121 and 21776148) and the Natural Science Foundation of Shandong Province (ZR2016BM07). Financial supports from the Qingdao Special Research Foundation of Science and Technology (16-6-2-29nsh) are also gratefully acknowledged.

\section{REFERENCES}

(1) Zhang, Y.; Li, B.; Ma, S. Dual functionalization of porous aromatic frameworks as a new platform for heterogeneous cascade catalysis. Chem. Commun. 2014, 50, 8507-8510.

(2) Xia, F.; Pan, M.; Mu, S.; Malpass-Evans, R.; Carta, M.; McKeown, N. B.; Attard, G. A.; Brew, A.; Morgan, D. J.; Marken, F. Polymers of intrinsic microporosity in electrocatalysis: Novel pore rigidity effects and lamella palladium growth. Electrochim. Acta 2014, 128, 3-9.

(3) Li, Y.; Bi, S.; Liu, F.; Wu, S.; Hu, J.; Wang, L.; Liu, H.; Hu, Y. Porosity-induced emission: exploring color-controllable fluorescence of porous organic polymers and their chemical sensing applications. $J$. Mater. Chem. C 2015, 3, 6876-6881.

(4) Kaleeswaran, D.; Vishnoi, P.; Murugavel, R. [3+3] Imine and [small beta]-ketoenamine tethered fluorescent covalent-organic frameworks for $\mathrm{CO} 2$ uptake and nitroaromatic sensing. J. Mater. Chem. C 2015, 3, 7159-7171.

(5) Arab, P.; Rabbani, M. G.; Sekizkardes, A. K.; İslamoğlu, T.; ElKaderi, H. M. Copper(I)-catalyzed synthesis of nanoporous azo-linked polymers: impact of textural properties on gas storage and selective carbon dioxide capture. Chem. Mater. 2014, 26, 1385-1392.

(6) Kim, S.; Lee, Y. M. Rigid and microporous polymers for gas separation membranes. Prog. Polym. Sci. 2015, 43, 1-32.

(7) Fang, Q.; Wang, J.; Gu, S.; Kaspar, R. B.; Zhuang, Z.; Zheng, J.; Guo, H.; Qiu, S.; Yan, Y. 3D porous crystalline polyimide covalent organic frameworks for drug delivery. J. Am. Chem. Soc. 2015, 137, $8352-8355$.

(8) Bai, L.; Phua, S. Z. F.; Lim, W. Q.; Jana, A.; Luo, Z.; Tham, H. P.; Zhao, L.; Gao, Q.; Zhao, Y. Nanoscale covalent organic frameworks as smart carriers for drug delivery. Chem. Commun. 2016, 52, 4128-4131.

(9) Prathap, A.; Sureshan, K. M. Organogelator-cellulose composite for practical and eco-friendly marine oil-spill recovery. Angew. Chem., Int. Ed. 2017, 56, 9405-9409.
(10) Ren, C.; Shen, J.; Chen, F.; Zeng, H. Rapid room-temperature gelation of crude oils by a wetted powder gelator. Angew. Chem., Int. Ed. 2017, 129, 3905-3909.

(11) Guterman, L. Exxon Valdez Turns 20. Science 2009, 323, 15581559.

(12) Acosta-González, A.; Rosselló-Móra, R.; Marqués, S. Characterization of the anaerobic microbial community in oil-polluted subtidal sediments: aromatic biodegradation potential after the Prestige oil spill. Environ. Microbiol. 2013, 15, 77-92.

(13) Parsons, M. L.; Morrison, W.; Rabalais, N. N.; Turner, R. E.; Tyre, K. N. Phytoplankton and the Macondo oil spill: A comparison of the 2010 phytoplankton assemblage to baseline conditions on the Louisiana shelf. Environ. Pollut. 2015, 207, 152-160.

(14) Aurell, J.; Gullett, B. K. Aerostat sampling of PCDD/PCDF emissions from the Gulf oil spill in situ burns. Environ. Sci. Technol. 2010, 44, 9431-9437.

(15) Gupta, D.; Sarker, B.; Thadikaran, K.; John, V.; Maldarelli, C.; John, G. Sacrificial amphiphiles: Eco-friendly chemical herders as oil spill mitigation chemicals. Sci. Adv. 2015, 1, No. e1400265.

(16) Ruan, C.; Ai, K.; Li, X.; Lu, L. A superhydrophobic sponge with excellent absorbency and flame retardancy. Angew. Chem., Int. Ed. 2014, 53, 5556-5560.

(17) Sabir, S. Approach of cost-effective adsorbents for oil removal from oily water. Crit. Rev. Environ. Sci. Technol. 2015, 45, 1916-1945.

(18) Liang, C. Composite absorbent particles for drying an emulsions. U.S. Patent 20,170,036,189A1. 2016.

(19) Liang, C.; Liu, Q.; Xu, Z. Dewatering Bitumen emulsions using interfacially active organic composite absorbent particles. Energy Fuels 2016, 30, 5253-5258.

(20) Ding, S.-Y.; Wang, W. Covalent organic frameworks (COFs): from design to applications. Chem. Soc. Rev. 2013, 42, 548-568.

(21) Xu, Y.; Jin, S.; Xu, H.; Nagai, A.; Jiang, D. Conjugated microporous polymers: design, synthesis and application. Chem. Soc. Rev. 2013, 42, 8012-8031.

(22) Huang, J.; Turner, S. R. Hypercrosslinked polymers: a review. Polym. Rev. 2018, 58, 1-41.

(23) Song, Q.; Cao, S.; Pritchard, R. H.; Ghalei, B.; Al-Muhtaseb, S. A.; Terentjev, E. M.; Cheetham, A. K.; Sivaniah, E. Controlled thermal oxidative crosslinking of polymers of intrinsic microporosity towards tunable molecular sieve membranes. Nat. Commun. 2014, 5, 4813.

(24) Yuan, Y.; Sun, F.; Li, L.; Cui, P.; Zhu, G. Porous aromatic frameworks with anion-templated pore apertures serving as polymeric sieves. Nat. Commun. 2014, 5, 4260.

(25) Liu, H.; Li, S.; Yang, H.; Liu, S.; Chen, L.; Tang, Z.; Fu, R.; Wu, D. Stepwise crosslinking: a facile yet versatile conceptual strategy to nanomorphology-persistent porous organic polymers. Adv. Mater. 2017, 29, 1700723.

(26) Zhang, C.-L.; Yu, S.-H. Nanoparticles meet electrospinning: recent advances and future prospects. Chem. Soc. Rev. 2014, 43, 44234448.

(27) Brown, T. D.; Dalton, P. D.; Hutmacher, D. W. Melt electrospinning today: An opportune time for an emerging polymer process. Prog. Polym. Sci. 2016, 56, 116-166.

(28) Liu, Y.; Luo, J.; Helleu, C.; Behr, M.; Ba, H.; Romero, T.; Hébraud, A.; Schlatter, G.; Ersen, O.; Su, D. S.; Pham-Huu, C. Hierarchical porous carbon fibers/carbon nanofibers monolith from electrospinning/CVD processes as a high effective surface area support platform. J. Mater. Chem. A 2017, 5, 2151-2162.

(29) Awenat, K. M.; Davis, P. J.; Moloney, M. G.; Ebenezer, W. A chemical method for the convenient surface functionalisation of polymers. Chem. Commun. 2005, 990-992.

(30) Yang, P.; Moloney, M. G. Surface modification of polymers with bis(arylcarbene)s from bis(aryldiazomethane)s: preparation, dyeing and characterization. RSC Adv. 2016, 6, 111276-111290.

(31) Yang, P.; Moloney, M. G.; Zhang, F.; Ji, W. Surface hydrophobic modification of polymers with fluorodiazomethanes. Mater. Lett. 2018, $210,295-297$. 
(32) Yang, P.; Wang, Y.; Lu, L.; Yu, X.; Liu, L. Surface hydrophobic modification of polyurethanes by diaryl carbene chemistry: Synthesis and characterization. Appl. Surf. Sci. 2018, 435, 346-351.

(33) Yang, P.; Moloney, M. G. Surface modification using crosslinking of diamine and a bis(diarylcarbene): synthesis, characterization, and antibacterial activity via binding hydrogen peroxide. RSC Adv. 2017, 7, 29645-29655.

(34) Griffiths, J.-P.; Maliha, B.; Moloney, M. G.; Thompson, A. L.; Hussain, I. Surface functional polymers by post-polymerization modification using diarylcarbenes: introduction, release and regeneration of hydrogen peroxide and bactericidal activity. Langmuir 2010, 26, 14142-14153.

(35) Wang, H.; Griffiths, J.-P.; Egdell, R. G.; Moloney, M. G.; Foord, J. S. Chemical functionalization of diamond surfaces by reaction with diaryl carbenes. Langmuir 2008, 24, 862-868.

(36) Aphaiwong, A.; Moloney, M. G.; Christlieb, M. Surface functional polymer library by post-polymerisation modification using diarylmethylenes: metal ligand catch and release. J. Mater. Chem. 2012, 22, 24627-24636.

(37) Hu, Z.; Shao, Q.; Moloney, M. G.; Xu, X.; Zhang, D.; Li, J.; Zhang, C.; Huang, Y. Nondestructive functionalization of graphene by surface-initiated atom transfer radical polymerization: an ideal nanofiller for poly(p-phenylene benzobisoxazole) fibers. Macromolecules 2017, 50, 1422-1429.

(38) Sartori, G.; Maggi, R.; Santacroce, V. Catalytic Friedel-Crafts acylation reactions. In Arene Chemistry: Reaction Mechanisms and Methods for Aromatic Compounds; Mortier, J., Ed.; John Wiley \& Sons, Inc: Hoboken, 2015; pp 59-82.

(39) Sander, W.; Bucher, G.; Wierlacher, S. Carbenes in matrixes: spectroscopy, structure, and reactivity. Chem. Rev. 1993, 93, 15831621.

(40) Ren, C.; Ng, G. H. B.; Wu, H.; Chan, K.-H.; Shen, J.; Teh, C.; Ying, J. Y.; Zeng, H. Instant room-temperature gelation of crude oil by chiral organogelators. Chem. Mater. 2016, 28, 4001-4008.

(41) Yao, H.; Yang, L.-P.; He, Z.-f.; Li, J.-R.; Jiang, W. A phaseselective, bis-urea organogelator with a curved bis-naphthalene core. Chin. Chem. Lett. 2017, 28, 782-786.

(42) Wu, J.; Wang, N.; Wang, L.; Dong, H.; Zhao, Y.; Jiang, L. Electrospun porous structure fibrous film with high oil adsorption capacity. ACS Appl. Mater. Interfaces 2012, 4, 3207-3212.

(43) Zhao, J.; Xiao, C.; Xu, N. Evaluation of polypropylene and poly (butylmethacrylate-co-hydroxyethylmethacrylate) nonwoven material as oil absorbent. Environ. Sci. Pollut. Res. 2013, 20, 4137-4145.

(44) Jiang, G.; Hu, R.; Xi, X.; Wang, X.; Wang, R. Facile preparation of superhydrophobic and superoleophilic sponge for fast removal of oils from water surface. J. Mater. Res. 2013, 28, 651-656.

(45) Chu, Y.; Pan, Q. Three-dimensionally macroporous $\mathrm{Fe} / \mathrm{C}$ nanocomposites as highly selective oil-absorption materials. ACS Appl. Mater. Interfaces 2012, 4, 2420-2425.

(46) Wang, C.-F.; Lin, S.-J. Robust superhydrophobic/superoleophilic sponge for effective continuous absorption and expulsion of oil pollutants from sater. ACS Appl. Mater. Interfaces 2013, 5, 88618864.

(47) Liu, H.-d.; Liu, Z.-y.; Yang, M.-b.; He, Q. Surperhydrophobic polyurethane foam modified by graphene oxide. J. Appl. Polym. Sci. 2013, 130, 3530-3536.

(48) Lei, W.; Portehault, D.; Liu, D.; Qin, S.; Chen, Y. Porous boron nitride nanosheets for effective water cleaning. Nat. Commun. 2013, 4, 1777.

(49) Zhang, X.; Geng, T.; Guo, Y.; Zhang, Z.; Zhang, P. Facile fabrication of stable superhydrophobic $\mathrm{SiO} 2$ /polystyrene coating and separation of liquids with different surface tension. Chem. Eng. J. 2013, 231, 414-419.

(50) Zhou, X.; Zhang, Z.; Xu, X.; Guo, F.; Zhu, X.; Men, X.; Ge, B. Robust and durable superhydrophobic cotton fabrics for oil/water separation. ACS Appl. Mater. Interfaces 2013, 5, 7208-7214. 